\title{
aFieldWork - an Android app for offline recording of geological information and data display
}

\author{
Martin Hansen, Matti Nelleman Petersen, Thomas F. Kokfelt and Bo Møller Stensgaard
}

Since 2008 the Geological Survey of Denmark and Greenland (GEUS) has used handheld devices (Personal Digital Assistents PDAs) for collecting field data in a digital format. Since PDAs are becoming obsolete and new device technology with improved functionality is available, it was decided to develop an Android-based application (app) that can be used by many mobile telephones and to test this on different devices during field work in Greenland.

The main objectives of the system are to provide field geologists working in remote areas without internet access with a quick and efficient way of: (1) recording information on a geological locality in digital format, (2) displaying existing digital geodata on maps, (3) securing a consistent way of reporting data and (4) transferring data quickly and efficiently to the central databases and GIS environments once the field teams return from their field work.

With the handheld device and the app (the system) the user collects data much in the same way as it was previously done using the modified GanFeld software with the PDAs (Schlatter et al. 2010), but with improved functions and a more user-friendly interface. The development of the system was guided by the following prerequisites:

- the device has to be relatively robust, lightweight, small and easy to handle,

- the device has to be easily replaceable and relatively cheap,

- the device includes an internal GPS and camera of sufficient quality with low power consumption; recharging must be with solar panels, or a mobile generator,

- the device must have backup, both internal (SD memory card) and external (laptop) during field work,

the app must be independent of mobile networks and must work offline,

- the app must store data in a well-structured format allowing for easy transfer to other GIS environments,

- the system should allow for both data collection and display in the field, and editing of data in the field camp,

- new data as well as existing geodata and maps (topographic data, geological maps, geochemical, geophysical, etc.) should be displayed together, and easily imported into the app,
- the system should have a simple, intuitive, user-friendly interface that is easy to operate in the field under difficult conditions.

The decision to develop an app for the Android platform was based on (1) the wide variety of relatively cheap Android devices available in many different sizes (including several robust versions), (2) the Java development language runs on a Windows platform, the main development platform used at GEUS, and (3) the possibility to store data on a SD removable memory card that can easily be transferred to a laptop or PC in the field camps.

\section{The aFieldWork app}

The app was designed to require as little typing as possible. This was done to make digital capture of field data as easy and efficient as possible. The app is database-driven meaning that data have to be entered in a structured and consistent fashion. Although some of the data entry is compulsory and is entered via scrollable, drop-down lists of pre-defined and fixed content, other information is optional and includes free-text entry. The mandatory entry points and the predefinition of selection lists also induce the field geologists to describe and classify localities and geological features in a consistent way.

The first version of the app was a prototype allowing for sufficient collection of information about localities, lithology, samples, structures and photographs. The system was further developed and refined based on field tests of the prototype in Greenland.

\section{Online and offline maps}

Two different map types are implemented in the app. The user can choose to use (1) online maps from OpenStreetMap (if connected to the internet), or (2) pre-loaded OpenStreetMap or a series of pre-loaded maps stored in a database. The last option is by far the most advanced and requires considerable preparation, but provides the most flexibility including custom-drawn maps at different zoom levels (scales) as well as various overlays of other data types (Fig. 1). 


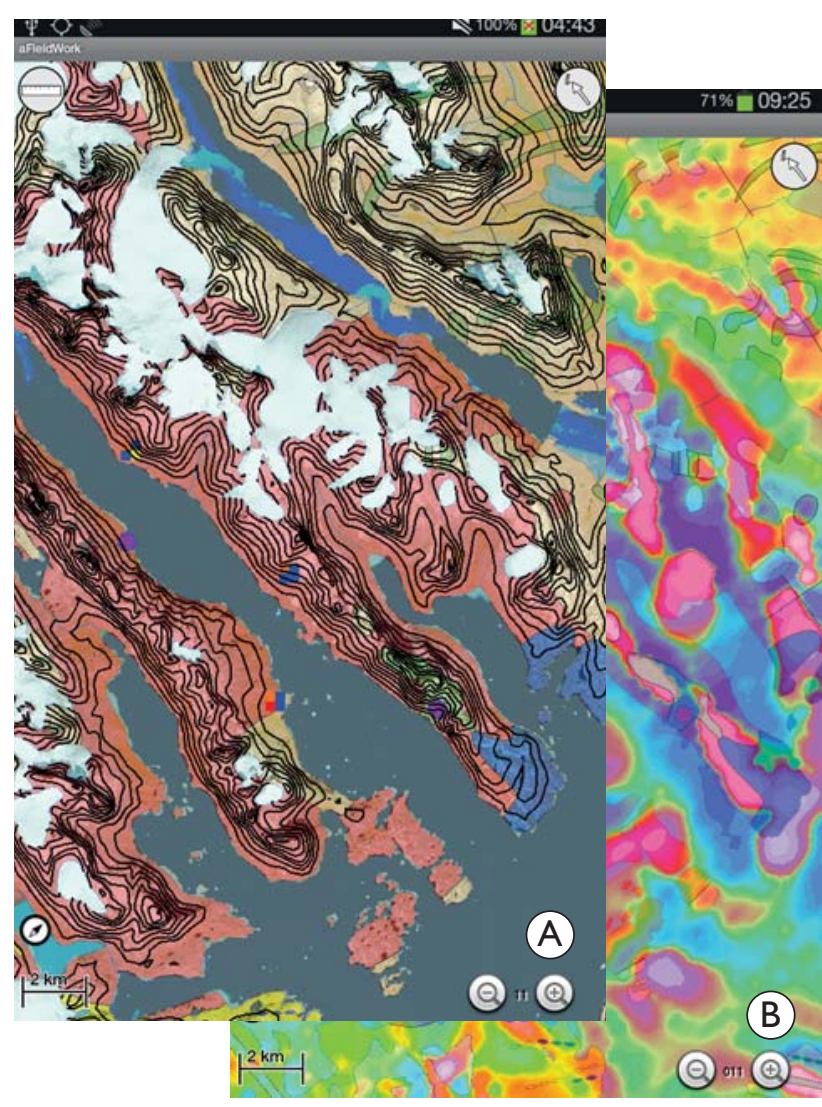

Fig. 1. A: Geological (1:500 000) map with layers of topographic contours and various locality point symbols. B: The same geographical area with an overlay of the analytical signal of the total magnetic intensity on top of the geological map.

All map types show and create geological localities as symbols. As long as the GPS is on, a colour-coded indicator shows each locality and the type of information which is attached to the locality. The user can make drawings and queries about spatial data, such as contours, trend lines and former localities. From the map a click on any locality or point will display attached information which can then be edited. While the map is displayed on the screen the user's position and walking direction will be shown. It is also possible to create points on the map that are not related to geological observations, a function useful for planning reconnaissance missions or drop-offs in the field by helicopter or boat. The user can make distance measurements on the maps and quickly obtain the coordinates of any point.

\section{How to enter data}

All data entries are geo-referenced via geographical co-ordinates taken from an internal GPS in the Android device together with information about its accuracy. The sample numbering follows GEUS' sample numbering system. The user may prompt manual text entries, for e.g. longer descriptions of localities, rocks and samples. For example, the data entry point, or menu, for samples will not appear before the user selects the 'sample button'. This ensures a smooth and efficient data entry without interference of unnecessary information and menus. The selection lists are in many cases designed so that the most frequently used entries are listed first and can be tailor-made before field use for special requirements.

'Localities'. A locality is created by tapping the 'New Locality' button on the start-up screen of the aFieldWork app (Fig. 2). The user is then prompted for a description of the locality (not mandatory). The co-ordinates are displayed as decimal degree, degree decimal minutes or converted to UTM coordinates, along with their GPS accuracy and time of creation. The locality is automatically stored and provided with a unique locality ID which is composed of the year, the unique GEUS initials of the geologist and the number of the locality, e.g. $14 \mathrm{smw} 003$ for the third locality of the geologist smw in the year 2014.

From the locality screen it is possible to add free text notes or to open entry points for 'Earth Materials', 'Samples', 'Structure' or 'Photos'. Each entry point has been colour-coded to facilitate identification (Fig. 2).

'Earth Materials' (lithology). The entry point 'Earth Materials' (Fig. 3) refers to information about the lithologies found at a given locality. This information is entered from a predefined, hierarchical system of 'Rock Class', 'Rock Type' and subsequently 'Rock Name' allowing a narrowing down to a few items with a few taps on the screen. The classification of the 'Earth Materials' (the rocks) follows classification schemes suggested by the International Union of Geological Sciences (IUGS). It is possible to omit a rock name at 'Earth Materials'. Extra information, such as colour, metamorphic grade, mineralogy (as 'common', 'alterations' and 'ore' minerals) and fossils can be entered. This information is mostly entered via predefined selection lists. Once the 'Earth Materials' data are entered it can be saved, not only to the locality, but also to a Quick list ('quiklst'). This makes it easy to add information from this Quick list to additional localities with the same 'Earth Material' information.

'Samples'. At the entry point 'Sample' the user can number and register information on particular rock samples collected in the field. The rock samples must be connected to an 


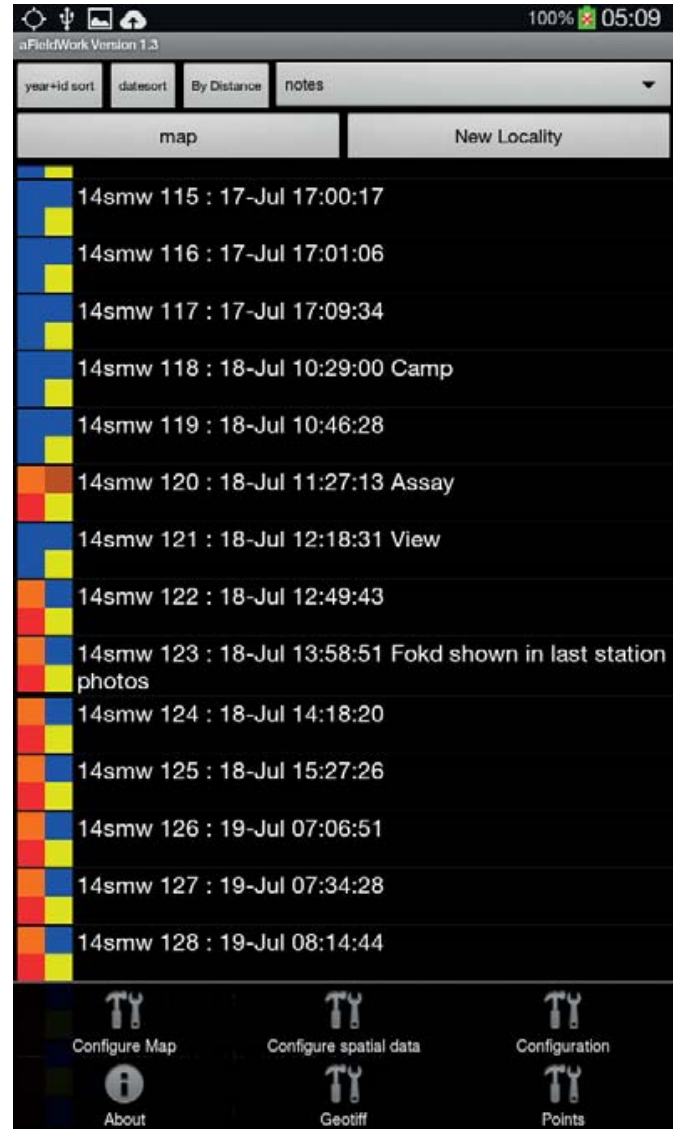

Fig. 2. The main screen of the app showing a list of localities with colour code added for easy reference: 'Earth Materials' (lithology), 'Samples', 'Structure' measurements or 'Photos'.

'Earth Material' entry and cannot be saved without this critical information. The 'Earth Material' entry can either be an existing one or can be created when the 'Sample' entry point is selected. The sample is automatically assigned a sample number (by default one increment above the previous sample number). A sample type and a sample purpose can be selected from drop-down lists and a free text can also be entered.

Structural measurements. At the entry point 'Structure' the user can digitally capture information on a structural measurement. Like rock samples, structural observations must be connected to an 'Earth Material'.

Structural class (linear or planar), type (e.g. fault, shear zone for a planar class) and detail (e.g. generation for a foliation) can be selected from drop-down lists. Measurements made by a hand-held compass can be entered as numbers using a keyboard or by two sliding bars.

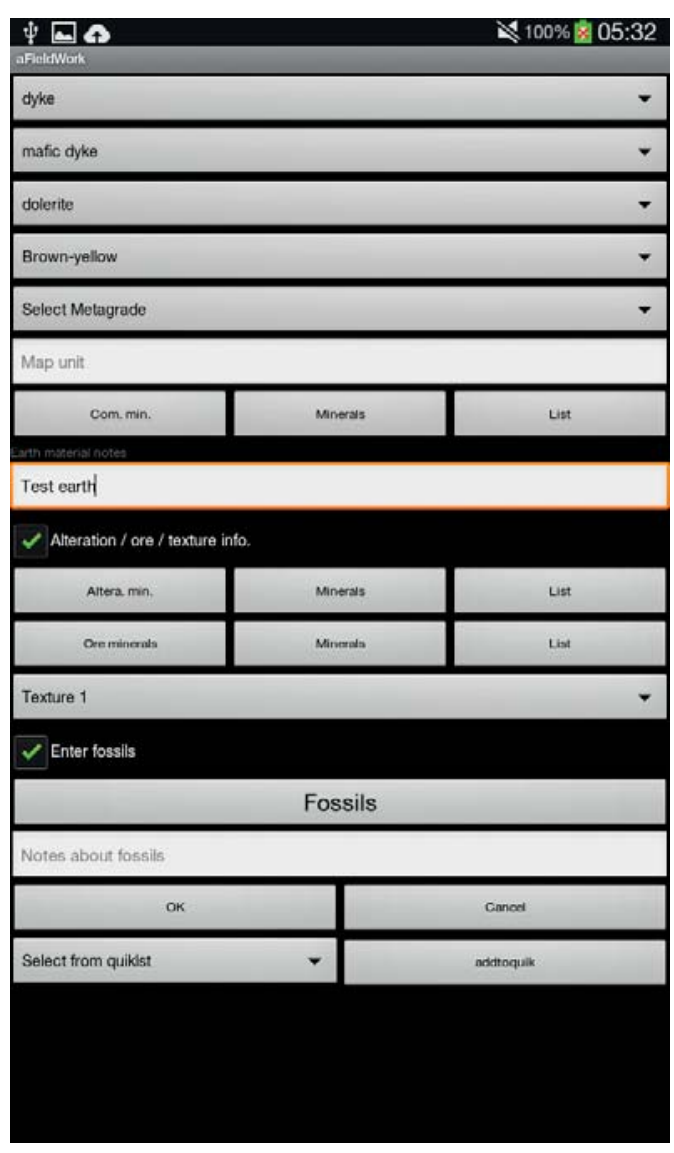

Fig. 3. The 'Earth Material' (lithology) data entry screen.

'Photos'. Photographs can be taken with the internal camera, in which case there is no need for data entry besides an optional free text description of the photograph. If an external camera is used, the user can enter the first and last number of the photographs taken at a given locality. It is also possible to enter free text for the photograph taken with an external camera. Sketches and annotations can be added to the photographs taken by the internal camera. They will be saved as separate files leaving the original photograph intact.

\section{Behind the screen}

All data are stored in an SQLite database used in most small digital devices. It is a relatively advanced database system supporting foreign keys, triggers and (with an extension) spatial data. The back-end database structure ensures wellstructured, compatible data and facilitates data transfer to 
other databases. Because the predefined selection lists in aFieldWork app are based on the database queries it is easy to extend the system or to configure it to suit the geological requirements of specific areas.

Part of the application (app) is based on open source software. The database handling is based on the Android SQLite Manger (aSQLiteManger), the map is based on the Mapsforge library (Mapsforge) and the spatial functions on the SpatiaLite library (SpatiaLite).

\section{Future development}

A future update of the app could be improved by adding a number of new features:

- import of points from Google Earth,

- built in documentation of all 'Earth materials' and 'Structure' classes,

- an easy overview of all structural data by symbols,

- the possibility to choose a location on the map and be guided there,

- easy export of data for analysis and planning of field work in field camps,

- use of air-pressure sensors to improve altitude determinations,

- new information fields for registering the type of locality, e.g. geological locality, field camp, archaeological sites, emergency pick-up site, etc.,

- display of the paths taken during field work,

- display geo-referenced raster images (e.g. images of geophysical, geochemical and remote sensing data) which will most likely be based on the RasterLite library (RasterLite).

Future versions of the app will enable the user to handle other sample types, e.g. stream sediments. It will be possible to develop and add new modules and functions including improved tools for importing and extracting data and maps.

\section{Conclusions}

Compared to the previous PDA-based system, data entry in aFieldWork app is much easier and faster with the new Android system. The design is simpler and more intuitive. The presence of a real database system and look-up tables on the devices ensures data integrity. It also allows swift transfer of data to central databases after field work. The SQLite databases are binary and compatible between different platforms so an SQLite database generated on an Android device can simply be moved to a Windows computer and used from this without any conversion.

The Android system is easy to configure and the large open-source community makes a lot of free software available.

GEUS has successfully used small digital handheld devices and software for digital data capture and observations on localities, rocks, samples, etc. in Greenland for a number of years. With the development of the new aFieldWork app, GEUS has modernised and improved this important field work tool for the geologist.

\section{Acknowledgements}

The Android system was tested during field work in South-East Greenland by participants in the SEGMENT 2012. The participants in these field parties provided valuable input and comments to the system which are greatly appreciated. Thanks to the two referees, Thorsten Nagel and Denis M. Schlatter, who contributed with valuable suggestions which greatly improved the manuscript.

\section{References}

Schlatter, D.M., Buller, G., Larsen, U. \& Stensgaard, B.M. 2010: Digital field data capture: the Geological Survey of Denmark and Greenland experiences in Greenland. The Association of Applied Geochemistry, Explore 147, 2-14.

aSQLiteManger, RasterLite, SpatiaLite http://www.gaia-gis.it/gaia-sins/ spatialite-sql-4.4.0.html

Mapsforge https://github.com/mapsforge/mapsforge 